\title{
A microRNA-27a mimic sensitizes human oral squamous cell carcinoma HSC-4 cells to hyperthermia through downregulation of Hsp110 and Hsp90
}

\author{
AYAKO KARIYA $^{1 *}$, YUKIHIRO FURUSAWA $^{1 *}$, TATSUYA YUNOKI $^{1}$, \\ TAKASHI KONDO ${ }^{1}$ and YOSHIAKI TABUCHI ${ }^{2}$ \\ ${ }^{1}$ Department of Radiological Sciences, Graduate School of Medicine and Pharmaceutical Sciences, and \\ ${ }^{2}$ Division of Molecular Genetics Research, Life Science Research Center, University of Toyama, Toyama 930-0194, Japan
}

Received November 26, 2013; Accepted February 27, 2014

DOI: $10.3892 /$ ijmm.2014.1758

\begin{abstract}
Hyperthermia (HT) is an important modality in cancer treatment; however, the acquisition of thermal resistance in cancer cells due to the elevation of heat shock proteins (HSPs) makes HT less effective. Accumulating evidence suggests that microRNAs (miRNAs) play an important role in regulating cellular stress sensitivities, such as drug sensitivity and radio-sensitivity, in cancer cells. However, few studies have investigated the involvement of miRNAs in thermal sensitivity. The aim of this study was thus to investigate the contribution of miRNAs to the thermal sensitivity of human oral squamous cell carcinoma (OSCC) cells. When the HSC-2, HSC-3 and HSC-4 OSCC cell lines were treated with HT at $44^{\circ} \mathrm{C}$ for $60 \mathrm{~min}$, a significant increase in cell death was observed in HSC-2 and HSC-3 cells but not HSC-4 cells, suggesting that HSC-4 cells were thermally resistant under the present experimental conditions. Moreover, the expression levels of HSPs were most elevated in HSC-4 cells. When the basal expression levels of miRNAs were monitored using two different microarray systems in thermal-sensitive HSC-2 and HSC-3 cells and thermal-resistant HSC-4 cells, five miRNAs
\end{abstract}

Correspondence to: Dr Yoshiaki Tabuchi, Division of Molecular Genetics Research, Life Science Research Center, University of Toyama, 2630 Sugitani, Toyama 930-0194, Japan

E-mail: ytabu@cts.u-toyama.ac.jp

${ }^{*}$ Contributed equally

Abbreviations: CDS, coding sequences; FBS, fetal bovine serum; GAPDH, glyceraldehyde 3-phosphate dehydrogenase; HSPs, heat shock proteins; HT, hyperthermia; miRNAs, microRNAs; OSCC, oral squamous cell carcinoma; PBS, phosphate-buffered saline; PI, propidium iodide; qPCR, quantitative polymerase chain reaction; 3'-UTR, 3'-untranslated region

Key words: microRNA, miR-27a, hyperthermia, human oral squamous cell carcinoma that were differentially expressed were identified. Among these miRNAs, the expression level of miR-27a in HSC-4 cells was markedly reducec compared to the expression levels in HSC-2 and HSC-3 cells. Interestingly, treatment of HSC-4 cells with a miR-27a mimic oligonucleotide significantly enhanced HT-induced cell death. Furthermore, the miR-27a mimic oligonucleotide suppressed the elevation of the expression of Hsp90 and Hsp110 in HSC-4 cells, suggesting that these HSPs may be involved in a mechanism of thermal resistance. From these findings, we concluded that in OSCC cells, miR-27a may contribute to thermal sensitivity by modulating the HSP expression.

\section{Introduction}

Local cancer control is an important objective of primary treatment, and decreasing the rate of recurrence is also important for patients. Hyperthermia (HT) induced by heat stress is a promising approach for the treatment of various types of malignant tumor, and is mainly used in combination with radiation therapy and/or chemotherapy (1). The great advantage of HT therapy is that it is tolerable for the majority of patients without severe toxicity. A better understanding of the mechanisms underlying its effects may provide important information for clinical HT therapy; thus, the biological effects have also been investigated for more than a decade. However, the heat sensitivities of cancer cells vary widely because of the differences in intrinsic heat sensitivity and resistance development. Since thermal-resistant cancer cells reduce the therapeutic effects of HT treatment, control of thermal resistance is a substantial clinical problem.

The mechanism of thermal resistance in most cancer cells involves the elevation of heat shock proteins (HSPs). Mammalian HSPs have been classified according to their molecular weights: Hsp110, Hsp90, Hsp70 and small HSPs (15-30 kDa) (2). HSPs are conserved proteins that are produced to protect cells from stress-induced damage by assisting the correct folding of nascent and stress-accumulated misfolded proteins, and by preventing their aggregation $(3,4)$. Cancer cells must expand their metabolic and signal transduction pathways, thereby becoming dependent on proteins, including 
stress-inducible HSPs, that are dispensable for the survival of normal cells. Therefore, the cytoprotective functions of HSPs mentioned above are necessary to maintain the survival of cancer cells (5). The expression and/or activity of HSPs are abnormally high in cancer cells and further increased after a variety of death stimuli, including HT (6).

microRNAs (miRNAs) are endogenous, evolutionarily conserved small (18-22 nucleotides) non-coding RNAs that have been shown to regulate gene expression post-transcriptionally (7). Currently, miRNAs are known to regulate the expression of their target genes by suppressing mRNA translation and/or degrading target mRNA transcription $(8,9)$. Due to their highly pleiotropic nature, each miRNA has the potential to regulate hundreds or even thousands of protein-coding RNA transcripts, and thus miRNAs are potentially capable of influencing many different molecules. Previous studies (8-10) have revealed that hundreds of miRNAs are found in the human genome and are critical in various important biological processes, including cell growth, proliferation, apoptosis and tumorigenesis. However, because of their complex mechanisms, the functions of miRNAs are not fully understood.

It has been suggested that miRNAs can functionally interact with a variety of environmental factors (11), including radiation (12), and HT (13). Results of recent studies have also shown that miRNAs play a role in the radiosensitivity of cancer cells $(14,15)$. However, few studies have been conducted specifically to investigate the involvement of miRNAs in thermal resistance in cancer cells. The aim of this study was to examine the effect of miRNAs on thermal resistance in human oral squamous cell carcinoma (OSCC) cell lines. The results showed that elevation of the expression level of miR-27a reinforces HT-induced cell death in thermal-resistant cells via a decrease in the expression levels of HSPs, especially Hsp110 and Hsp90.

\section{Materials and methods}

Cell culture and HT treatment. The human OSCC cell lines HSC-2, HSC-3 and HSC-4 were obtained from the Human Science Research Resources Bank of the Japan Health Sciences Foundation (Tokyo, Japan). The cell lines were cultured in E-MEM medium (Wako Pure Chemical Industries, Ltd., Osaka, Japan) supplemented with $10 \%$ fetal bovine serum (FBS) at $37^{\circ} \mathrm{C}$ in humidified air with $5 \% \mathrm{CO}_{2}$. For the $\mathrm{HT}$ treatment, cells were cultured in 6- or 12-well plates for $72 \mathrm{~h}$ prior to HT treatment. The plates were sealed with Parafilm ${ }^{\circledR}$ and heated at $44^{\circ} \mathrm{C}$ for $60 \mathrm{~min}$ in a water bath. After HT treatment, the cells were incubated at $37^{\circ} \mathrm{C}$ for the indicated time periods until analysis (16).

Analysis of cell death. Cells were collected $24 \mathrm{~h}$ after HT treatment. For the detection of total cell death (apoptotic and necrotic cell death), the cells were washed with ice-cold phosphate-buffered saline (PBS) and treated with $2.5 \mu \mathrm{g} / \mathrm{ml}$ propidium iodide (PI) solution. For the detection of chromatin condensation, the cells were stained using a Nuclear-ID Green Chromatin Condensation kit (Enzo Life Sciences Inc., Farmingdale, NY, USA) according to the manufacturer's instructions. To obtain the distribution of cells in a sub-G1 phase of the cell cycle, the cells were fixed with $70 \%$ ice-cold ethanol, and subsequently treated with $0.25 \mu \mathrm{g} / \mathrm{ml}$ RNase A and $50 \mu \mathrm{g} / \mathrm{ml}$ PI. The samples were then run on an Epics XL flow cytometer (Beckman Coulter, Fullerton, CA, USA) (17).

Western blot analysis. Whole-cell extracts were prepared in RIPA lysis buffer containing a cocktail of protease inhibitors (Nacalai Tesque, Kyoto, Japan). The polyvinylidene difluoride membranes were incubated with the primary antibody at $4^{\circ} \mathrm{C}$ for $18 \mathrm{~h}$, and exposed to the peroxidaseconjugated secondary antibody at room temperature for $1 \mathrm{~h}$. Immunoreactive proteins were visualized by a luminescent image analyzer using a chemiluminescence detection system. Primary antibodies used were as follows: a rabbit polyclonal anti-Hsp110 antibody, a rat monoclonal anti-Hsp90 antibody, a mouse monoclonal anti-Hsp70 antibody, a mouse monoclonal anti-Hsp40 antibody (all from MBL, Nagoya, Japan), a rabbit polyclonal anti-caspase-3 antibody (Cell Signaling Technology, Danvers, MA, USA) or a mouse monoclonal anti-glyceraldehyde 3-phosphate dehydrogenase (GAPDH) antibody (Millipore Co., Temecula, CA, USA).

Detection of miRNAs. Total RNA containing small RNAs was extracted from the cultured cells using a miRNeasy Mini kit (Qiagen, Valencia, CA, USA) according to the manufacture's instructions and used for the global miRNA expression analysis. The quality of RNA was determined with an Agilent Bioanalyzer 2100 (Agilent Technologies, Inc., Santa Clara, CA, USA). The miRNA microarray profiling was performed using two miRNA array systems. One was the GeneChip ${ }^{\circledR}$ miRNA 2.0 array, which has unique probes for 15,644 mature miRNAs from miRBase and 2,202 probes for pre-miRNA hairpin sequences (Affymetrix, Inc., Santa Clara, CA, USA). Total RNA ( $1 \mu \mathrm{g})$ was labeled by poly(A) polymerase addition using a Genisphere FlashTag HSR kit following the manufacturer's instructions (Genisphere, Hatfield, PA, USA). Labeled RNA was hybridized to the miRNA 2.0 arrays. The arrays were washed and stained in a Fluidics Station 450, and image scanning was performed using an Affymetrix scanner. The other array system was Toray's 3D-Gene ${ }^{\mathrm{TM}}$ human miRNA array, which contains $\sim 1,700$ probes selected from miRBase (Toray Industries, Inc., Tokyo, Japan). Labeling, scanning and data mining were performed at the Toray Research Center (Toray Industries, Inc.).

Quantitative polymerase chain reaction ( $q P C R)$ assay. Total RNA was extracted from cells using an RNeasy total RNA extraction kit (Qiagen). qPCR was performed on a Real-Time qPCR system (Mx3000P; Agilent Technologies, Inc.) using SYBR Premix DimerEraser ${ }^{\mathrm{TM}}$ (Takara Bio, Inc., Shiga, Japan) according to the manufacturer's instructions. The reverse transcriptase reaction was carried out with total RNA by using a random 6 mers and an oligo(dT) primer (PrimeScript RT reagent kit; Takara Bio, Inc.). PCR primers were designed based on the database. GAPDH was used as a control for the normalization $(17,18)$.

miRNA transfection. The pre-miRNAs, hsa-miR-23a mimic, hsa-miR-27a-3p mimic/antisense, hsa-miR-30a antisense, has-miR-30c antisense, hsa-miR-203 antisense, and miScript inhibitor negative control were obtained from Qiagen. miRNA 

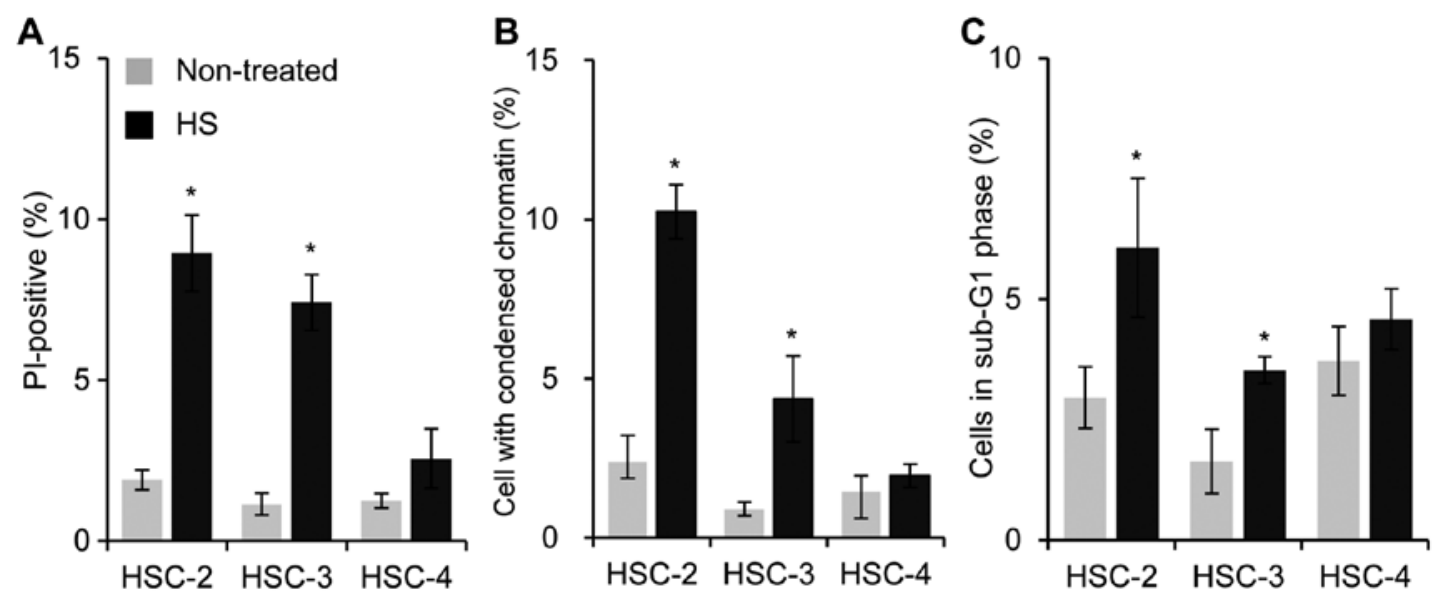

Figure 1. Effects of hyperthermia (HT) on the cell death in oral squamous cell carcinoma (OSCC) cells. HSC-2, HSC-3, and HSC-4 OSCC cell lines were treated with $\mathrm{HT}$ at $44^{\circ} \mathrm{C}$ for $60 \mathrm{~min}$ and then cultured at $37^{\circ} \mathrm{C}$ for $24 \mathrm{~h}$. (A) Total cell death (apoptotic and necrotic cell death), (B) chromatin condensation, and (C) sub-G1 phase of the cell cycle were measured. Gray columns, non-treated group; black columns, HT-treated group. Data are presented as the means \pm standard deviations (SDs) ( $\mathrm{n}=4)$. ${ }^{*} \mathrm{P}<0.05$ vs. each non-treated group.

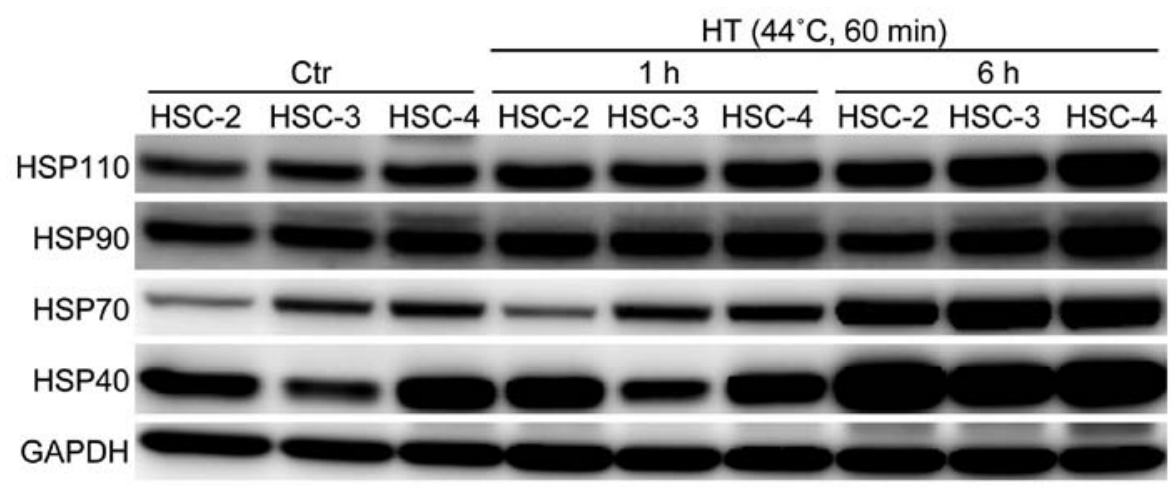

Figure 2. Measurement of the protein expression levels of heat shock proteins (HSPs) induced by hyperthermia (HT). Cells were exposed to HT at $44^{\circ} \mathrm{C}$ for $60 \mathrm{~min}$ and then cultured at $37^{\circ} \mathrm{C}$ for $24 \mathrm{~h}$. Protein expression levels of HSPs and glyceraldehyde 3-phosphate dehydrogenase (GAPDH) were determined by western blot analysis. Ctr, non-treated cells.

was transfected to cells using Lipofectamine ${ }^{\mathrm{TM}}$ RNAiMAX (Life Technologies Co., Grand Island, NY, USA) according to the manufacturer's instructions. Briefly, the cells were plated in 12-well plates in E-MEM medium supplemented with $10 \%$ FBS. After growth for 20-24 h, the cells were replaced in serum-reduced Opti-MEM (Life Technologies Co.) containing the Lipofectamine ${ }^{\mathrm{TM}}$ RNAiMax/miRNA complexes that were prepared $20 \mathrm{~min}$ before addition to the cells (forward transfection protocol). To decrease the cellular toxicity of the complexes, Opti-MEM containing the complexes was replaced with the complete culture medium $6 \mathrm{~h}$ after transfection. Forty-eight hours after transfection, the cells were exposed to HT.

Statistical analysis. Data are presented as the means \pm standard deviations (SDs). Differences between pairs of data sets were analyzed using the Student's t-test, with values of $\mathrm{P}<0.05$ considered to indicate statistically significant differences.

\section{Results}

Effects of HT on the cell death in OSCC cells. To evaluate the thermo-sensitivity of the HSC-2, HSC-3 and HSC-4 OSCC cell lines, the effects of HT on the cell death were examined using flow cytometry. Twenty-four hours after HT treatment $\left(44^{\circ} \mathrm{C}\right.$, $60 \mathrm{~min}$ ), the percentages of total cell death (apoptotic and necrotic dead cells) and condensed chromatin condensation (apoptotic cell death) were significantly increased in the HSC-2 and HSC- 3 cells but not in the HSC-4 cells (Fig. 1A and B). The measurement of the distribution of cells in a sub-G1 phase of the cell cycle, a marker for apoptosis, also supported the idea that HSC-2 and HSC-3 were more sensitive to HT than HSC-4 cells (Fig. 1C). The results suggested that $\mathrm{HT}$ induces cell death in HSC-2 and HSC-3 but not in HSC-4 cells. Therefore, HSC-4 cells were less sensitive to HT than the other two cell lines.

Induction of HSPs in OSCC cells by HT. Having established that the OSCC cell lines showed different levels of thermal sensitivity, we examined the expression patterns of HSPs in the OSCC cell lines by means of western blot analysis. The basal expression level of the Hsp70 protein was higher in HSC-3 and HSC-4 cells than in the HSC-2 cells (Fig. 2). On the other hand, the basal expression level of the $\mathrm{Hsp} 40$ protein was higher in the HSC-2 and HSC-4 cells than in the HSC-3 cells. In terms of the Hsp90 and Hsp110 proteins, HSC-4 cells 

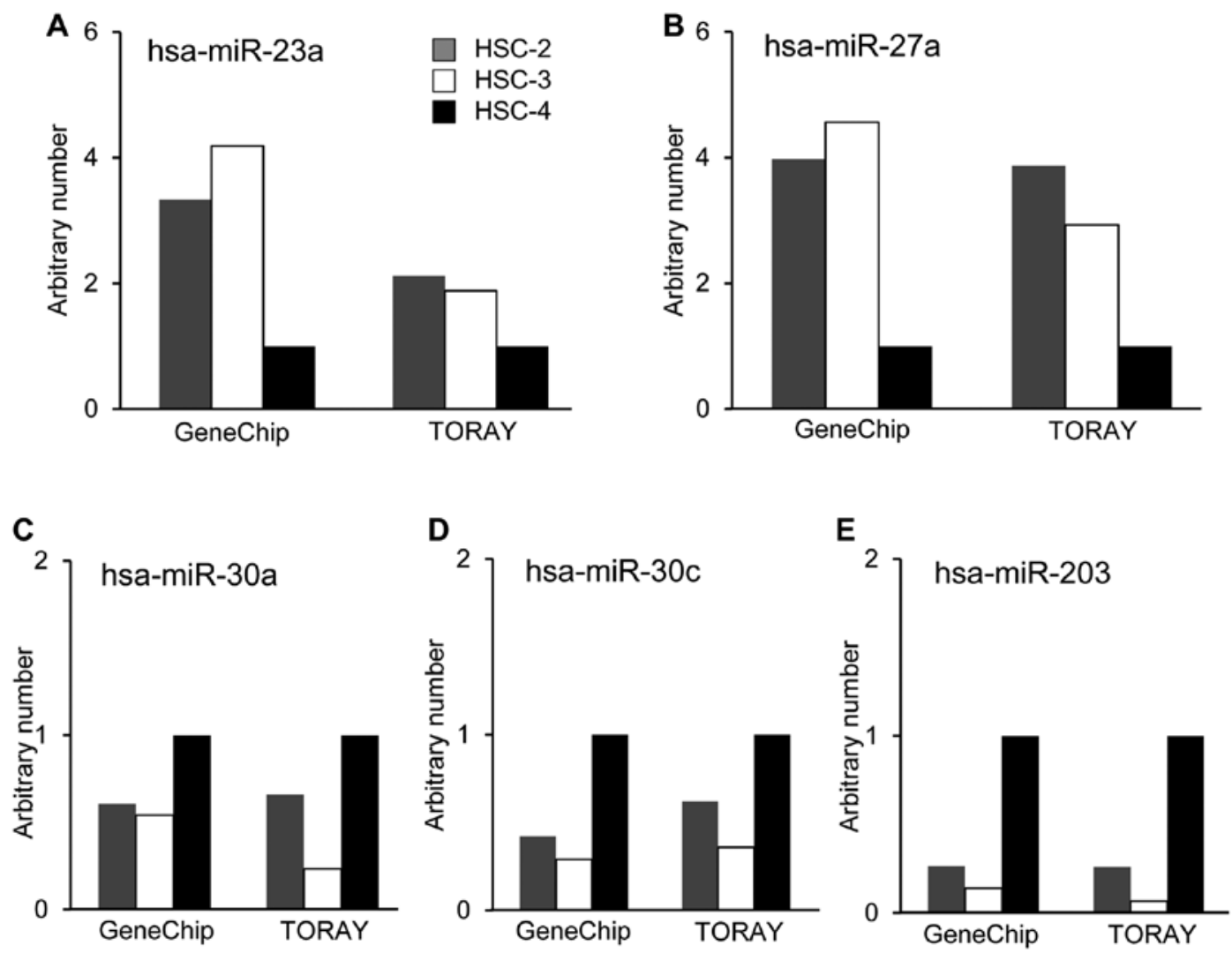

Figure 3. Comprehensive expression analysis for the detection of miRNAs that were differentially expressed in the oral squamous cell carcinoma (OSCC) cell lines. Two miRNA array systems, the GeneChip ${ }^{\circledR}$ miRNA 2.0 array and Toray's 3D-Gene ${ }^{\mathrm{TM}}$ human microRNA, were used to analyze the expression profiles of miRNAs in OSCC cells. (A and B) The expression levels of hsa-miR-23a and hsa-miR-27a in HSC-4 cells were lower than those of other cells. (C-E) hsa-miR-30a, hsa-miR-30c and hsa-miR-203 were preferentially expressed in HSC-4 cells compared with HSC-2 and HSC-3 cells. Gray columns, HSC-2 cells; white columns, HSC-3 cells; black columns, HSC-4 cells. An arbitrary number was determined by each miRNA expression level in HSC-4 cells as 1.0.

showed the highest expression levels of these HSPs among the three cell lines examined. After HT treatment, the protein expression levels of HSPs were increased in a time-dependent manner, and the induction rates of Hsp70 and Hsp40 were marked in all the cell lines. Notably, $6 \mathrm{~h}$ after HT exposure, the protein expression levels of Hsp110 and Hsp90 in HSC-4 cells were further increased and were the highest among the three cell lines (Fig. 2). Therefore, we hypothesized that the different protein expression patterns of HSPs may explain the differences in thermal sensitivity of the OSCC cells lines, especially the thermo-resistance of HSC-4 cells.

Expression profiles of miRNAs in OSCC cells. To identify miRNAs that were differentially expressed and associated with the thermal sensitivity, we utilized two different miRNA microarray systems provided by Affymetrix, Inc. and Toray Industries, Inc. The results showed marked differences in the miRNA expression patterns between the thermo-sensitive cell lines, HSC-2 and HSC-3 cells, and the thermo-resistant cell line, HSC-4, in the two systems. The basal expression levels of several miRNAs in HSC-4 cells were significantly different compared with those in HSC-2 and HSC-3 cells. The expression levels of hsa-miR-23a and hsa-miR-27a in HSC-4 cells were lower than those of other cells (Fig. 3A and B). On the other hand, hsa-miR-30a, hsa-miR-30c and hsa-miR-203 were preferentially expressed in HSC-4 cells compared with HSC-2 and HSC-3 cells (Fig. 3C-E).
Effects of miRNA mimic or antisense oligonucleotides on HT-induced cell death in HSC-4 cells. The two independent microarray systems clearly demonstrated that five miRNAs (miR-23a, miR-27a, miR-30a, miR-30c and miR-203) were candidate miRNAs for thermal sensitivity in OSCC cells. Therefore, we examined the effects of miRNA mimic or antisense oligonucleotides on HT-induced cell death. HSC-4 cells were treated with HT $48 \mathrm{~h}$ after being transfected with the oligonucleotide, and then cell death was evaluated $24 \mathrm{~h}$ after HT treatment. Treatments of HSC-4 cells with mimic oligonucleotides for miR-23a (20 nM) and miR-27a (20 nM) followed by HT significantly elevated cell death, with the mean percentages of cell death being 8.5 and $18.0 \%$, respectively (Fig. 4A and B). However, cell death was hardly enhanced by the treatment with a high concentration $(100 \mathrm{nM})$ of antisense oligonucleotide for miR-30, miR-30c or miR-203 in HT-exposed cells (data not shown). These results demonstrated that miR-23a and miR-27a may be involved in thermal sensitivity in HSC-4 cells.

Effects of the miR-27a mimic oligonucleotide on the protein expression of HSPs and cleavage of caspase-3 in HSC-4 cells under HT-treated conditions. We examined whether the protein expression of HSPs was influenced by treatment with the miR-27a mimic oligonucleotide in HSC-4 cells. The protein expression levels of Hsp110 and Hsp90 were significantly decreased in HSC-4 cells treated with the miR-27a 

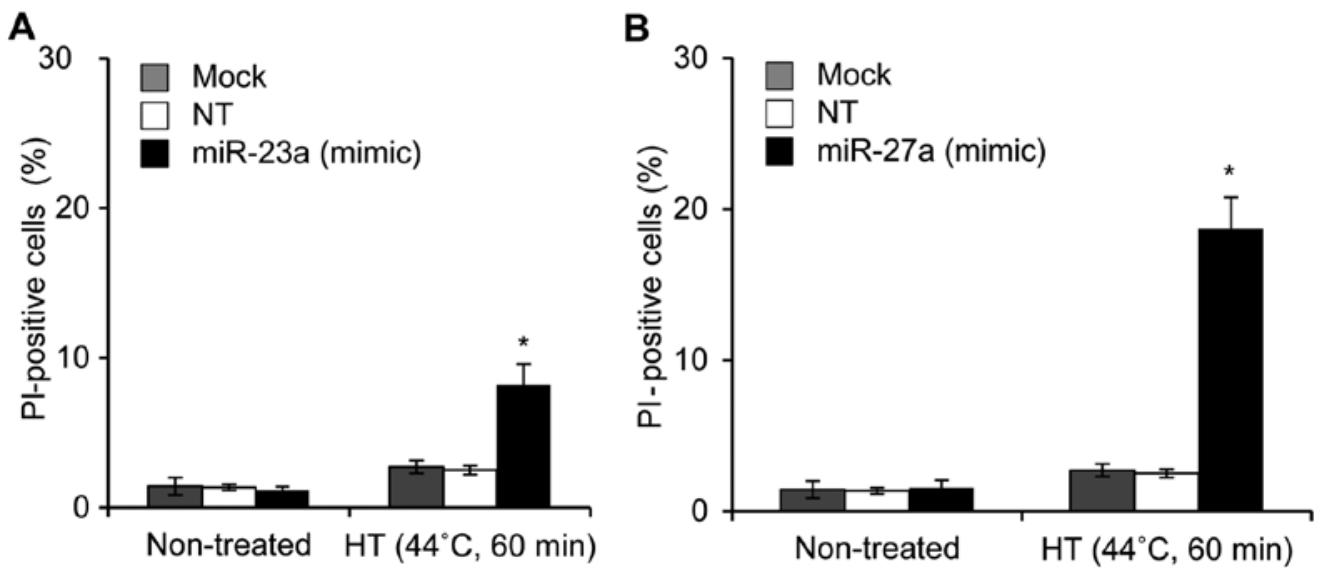

Figure 4. Effects of the microRNA mimic oligonucleotides on hyperthermia (HT)-induced cell death in HSC-4 cells. HSC-4 cells were transfected with (A) miR-23a mimic $(20 \mathrm{nM})$ or (B) miR-27a mimic $(20 \mathrm{nM})$ oligonucleotides. Forty-eight hours after transfection, the cells were treated with $\mathrm{HT}$ at $44^{\circ} \mathrm{C}$ for $60 \mathrm{~min}$ followed by culturing at $37^{\circ} \mathrm{C}$ for $24 \mathrm{~h}$. Cell death was monitored using propidium iodide staining. Gray columns, mock (transfection reagent only); white columns, NT (non-targeted miRNA control); black columns, each oligonucleotide. Data are presented as the means \pm standard deviations (SDs) ( $\mathrm{n}=3$ ). ${ }^{*} \mathrm{P}<0.05$ vs. each mock control.

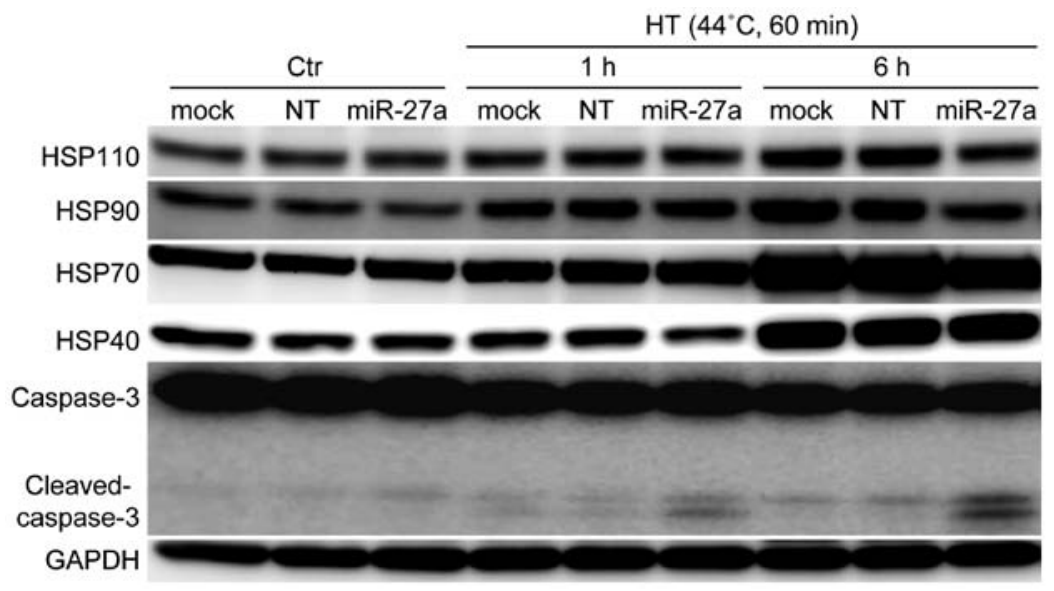

Figure 5. Effects of the miR-27a mimic oligonucleotide on the expression of heat shock proteins (HSPs) and cleavage of caspase-3 in HSC-4 cells under hyperthermia (HT)-treated conditions. HSC- 4 cells were exposed to HT at $44^{\circ} \mathrm{C}$ for $60 \mathrm{~min}$ after $48 \mathrm{~h}$ of transfection with the miR-27a mimic oligonucleotide $(20 \mathrm{nM})$ and then cultured at $37^{\circ} \mathrm{C}$ for 1 and $6 \mathrm{~h}$. Protein expression levels of HSPs, caspase-3 and glyceraldehyde 3-phosphate dehydrogenase (GAPDH) were determined by western blot analysis. Ctr, non-treated cells. Mock, transfection reagent only; NT, non-targeted miRNA control.
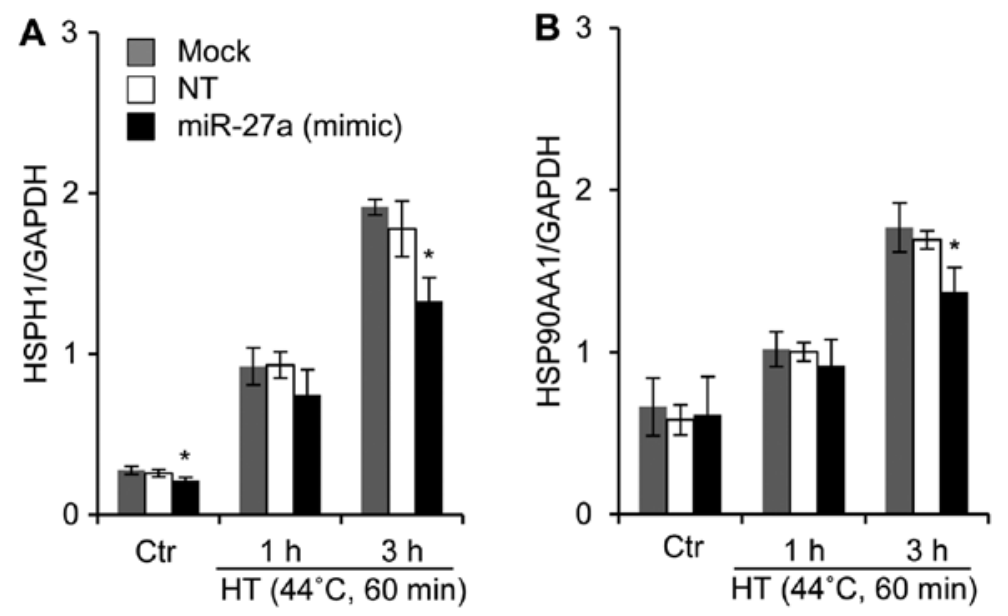

Figure 6. Effects of the miR-27a mimic oligonucleotide on mRNA expression of heat shock proteins (HSPs) in HSC-4 cells under hyperthermia (HT)-treated conditions. HSC- 4 cells were exposed to $\mathrm{HT}$ at $44^{\circ} \mathrm{C}$ for $60 \mathrm{~min} 48 \mathrm{~h}$ after transfection of the miR-27a mimic oligonucleotide (20 nM) and then cultured at $37^{\circ} \mathrm{C}$ for 1 and $3 \mathrm{~h}$. Quantitative polymerase chain reaction was performed with specific primers for (A) HSPH1 or (B) HSP90AA1. Each mRNA expression level was normalized to the expression level of glyceraldehyde 3-phosphate dehydrogenase (GAPDH). Gray columns, mock (transfection reagent only); white columns, NT (non-targeted miRNA control); black columns, miR-27a (miR-27a mimic oligonucleotide). Ctr, non-treated cells. Data are presented as the means \pm standard deviations (SDs) ( $=4)$. ${ }^{*} \mathrm{P}<0.05$ vs. each mock control. 
mimic oligonucleotide (20 $\mathrm{nM}$ ) under HT conditions (Fig. 5). Simultaneously, the oligonucleotide slightly decreased the expression levels of Hsp70 and Hsp40. A significant increase in caspase-3 cleavage, a marker of apoptosis, was observed in the cells following combination treatment with HT and oligonucleotide transfection. These results suggested that the increased cleavage of caspase-3 may be due to the decreased expression levels of HSPs, especially Hsp110 and Hsp90, which promote cancer cell survival $(19,20)$.

Effects of the miR-27a mimic oligonucleotide on the mRNA expression of HSPs in HSC-4 cells under HT-treated conditions. A bioinformatics-based approach was employed to predict the putative targets using the TargetScan program hosted by the Wellcome Trust Sanger Institute (21) and GGRNA hosted by DBCLS (22). We noted that one potential binding site was found in the 3'-untranslated region (3'-UTR) or amino acid coding sequences (CDS) of the human HSPH1 (Hsp110), HSP90AA1 (Hsp90), HSPA1A (Hsp70), HSPA1B (Hsp70), DNAJA1 (Hsp40), and DNAJB1 (HSp40) genes for human miR-27a. We also investigated whether a miR-27a mimic oligonucleotide would affect the mRNA expression levels of HSPs in HSC-4 cells. In the miR-27a mimic oligonucleotide-transfected cells, the mRNA expression levels of HSPH1 and HSP90AA1 were significantly reduced when the cells were exposed to HT (Fig. 6). By contrast, the oligonucleotide did not affect the mRNA expression of HSPA1A, HSPA1B, DNAJA1 and DNAJB1 under either regular or HT conditions (data not shown).

\section{Discussion}

Control of thermal resistance is one of the most important issues in HT therapy. It has been reported that miRNAs can play a critical role in cancer cells (23) by regulating cancer-related pathways such as the cell cycle control, DNA damage response and stress-sensitivity pathways $(23,24)$. Microarray expression data from a wide spectrum of cancers have provided evidence that aberrant miRNA expression is the rule rather than the exception in cancer (25). However, the resistance to heat stress in cancer cells remains largely unexplored. In the present study, we revealed that OSCC HSC-4 cells, the model of thermally resistant cells used in the present study, had characteristic patterns of miRNA expression compared with the thermal-sensitive HSC-2 and HSC-3 OSCC cell lines. Of the miRNAs expressed in this manner, miR-27a was most likely to be involved in the thermal resistance in HSC-4 cells, because its constitutive expression level was lower than the levels in thermal-sensitive OSCC cells. Notably, transfection of HSC-4 cells with a miR-27a mimic oligonucleotide elicited cell death under the HT conditions. These results suggest that the low expression level of miR-27a likely contributes to the mechanism of thermal resistance in OSCC HSC-4 cells.

As for the targets of miR-27a, we focused on the HSP family genes. miR-27a partially shares the seed sequence (26) to bind to the 3'-UTR or the amino acid CDS region of HSP family genes. In general, miRNAs modulate the gene expression in mammalian cells by base pairing to complementary sites in the 3'-UTR of their target miRNAs. However, results of a recent study showed that miRNAs can regulate target
miRNAs by binding to the CDS region (27). In the present study, the expression levels of Hsp110 and Hsp90 significantly decreased when HSC-4 cells were transfected with the miRNA-27a mimic oligonucleotide under the HT conditions (Fig. 5), despite the low homology of the seed sequence to these 3'-UTR regions. Additionally, these CDS regions have partial homology to the miR-27a seed. Notably, the miR-27a mimic oligonucleotide significantly suppressed the expression of Hsp110 and Hsp90, not only at the levels of translation, but also transcription. The detailed mechanism underlying the control of gene expression via the CDS regions has not yet been identified, although the homology between the miR-27a seed sequence and CDS regions of Hsp110 and Hsp90 genes may be important for the regulation of those expressions.

It is generally recognized that HSPs confer substantial thermal resistance to cancer cells (6). Hsp110, one of the earliest HSPs described in mammalian cells, plays an important role as a chaperone under stress conditions $(19,28)$ and participates in cellular thermal resistance (29). In addition, it has been recently demonstrated that the expression level of Hsp110 is elevated in highly metastatic colon cancer cell lines, and is correlated with advanced clinical stages (30). Hsp90 is also a molecular chaperone protein and is crucially involved in the function and stability of many oncogene products and cell-signaling molecules $(31,32)$. Functional inhibition of Hsp90 also appears to enhance the sensitivity to HT on the incidence of cell death (33). Overexpression of Hsp90 was frequently observed in cancer cells, and, therefore, the pharmacological inhibition of Hsp90 is an attractive strategy for cancer therapy (34).

In conclusion, miR-27a may contribute to the thermal sensitivity of OSCCs, presumably through regulation of the expression of Hsp110 and Hsp90. Our data supports a model in which miR-27a may overcome thermal resistance and serve as a predictive marker of thermal sensitivity.

\section{Acknowledgements}

This study was supported in part by a Grant-in-Aid for the Challenging Exploratory Research (23650303) and a Grant-in-Aid for Scientific Research B (24310046) from the Japan Society for the Promotion of Science. We would like to thank Dr Ryohei Ogawa for helpful advice.

\section{References}

1. Hildebrandt B, Wust P, Ahlers O, et al: The cellular and molecular basis of hyperthermia. Crit Rev Oncol Hematol 43: 33-56, 2002.

2. Vos MJ, Hageman J, Carra S and Kampinga HH: Structural and functional diversities between members of the human HSPB, HSPH, HSPA, and DNAJ chaperone families. Biochemistry 47: 7001-7011, 2008.

3. Minton KW, Karmin P, Hahn GM and Minton AP: Nonspecific stabilization of stress-susceptible proteins by stress-resistant proteins: a model for the biological role of heat shock proteins. Proc Natl Acad Sci USA 79: 7107-7111, 1982.

4. Lanneau D, Brunet M, Frisan E, Solary E, Fontenay M and Garrido C: Heat shock proteins: essential proteins for apoptosis regulation. J Cell Mol Med 12: 743-761, 2008.

5. Ciocca DR and Calderwood SK: Heat shock proteins in cancer: diagnostic, prognostic, predictive, and treatment implications. Cell Stress Chaperones 10: 86-103, 2005.

6. Calderwood SK, Khaleque MA, Sawyer DB and Ciocca DR: Heat shock proteins in cancer: chaperones of tumorigenesis. Trends Biochem Sci 31: 164-172, 2006. 
7. Filipowicz W, Bhattacharyya SN and Sonenberg N: Mechanisms of post-transcriptional regulation by microRNAs: are the answers in sight? Nat Rev Genet 9: 102-114, 2008.

8. Meltzer PS: Cancer genomics: small RNAs with big impacts. Nature 435: 745-746, 2005.

9. Krol J, Loedige I and Filipowicz W: The widespread regulation of microRNA biogenesis, function and decay. Nat Rev Genet 11: 597-610, 2010

10. Moss EG: MicroRNAs: hidden in the genome. Curr Biol 12: R138-R140, 2002

11. Wang $\mathbf{J}$ and Cui Q: Specific roles of microRNAs in their interactions with environmental factors. J Nucleic Acids 2012: 978384, 2012.

12. Niemoeller OM, Niyazi M, Corradini S, et al: MicroRNA expression profiles in human cancer cells after ionizing radiation. Radiat Oncol 6: 29, 2011

13. Wilmink GJ, Roth CL, Ibey BL, et al: Identification of microRNAs associated with hyperthermia-induced cellular stress response. Cell Stress Chaperones 15: 1027-1038, 2010.

14. Gwak HS, Kim TH, Jo GH, et al: Silencing of microRNA-21 confers radio-sensitivity through inhibition of the PI3K/AKT pathway and enhancing autophagy in malignant glioma cell lines. PLoS One 7: e47449, 2012.

15. Chistiakov DA and Chekhonin VP: Contribution of microRNAs to radio- and chemoresistance of brain tumors and their therapeutic potential. Eur J Pharmacol 684: 8-18, 2012.

16. Tabuchi Y, Wada S, Furusawa Y, Ohtsuka K and Kondo T: Gene networks related to the cell death elicited by hyperthermia in human oral squamous cell carcinoma HSC-3 cells. Int J Mol Med 29: 380-386, 2012.

17. Kariya A, Tabuchi Y, Yunoki T and Kondo T: Identification of common gene networks responsive to mild hyperthermia in human cancer cells. Int J Mol Med 32: 195-202, 2013.

18. Tabuchi Y, Furusawa Y, Kariya A, Wada S, Ohtsuka K and Kondo T: Common gene expression patterns responsive to mild temperature hyperthermia in normal human fibroblastic cells. Int J Hyperthermia 29: 38-50, 2013.

19. Hosaka S, Nakatsura T, Tsukamoto H, Hatayama T, Baba H and Nishimura Y: Synthetic small interfering RNA targeting heat shock protein 105 induces apoptosis of various cancer cells both in vitro and in vivo. Cancer Sci 97: 623-632, 2006.

20. Xu W and Neckers L: Targeting the molecular chaperone heat shock protein 90 provides a multifaceted effect on diverse cell signaling pathways of cancer cells. Clin Cancer Res 13: $1625-1629,2007$
21. Plaisance-Bonstaff $\mathrm{K}$ and Renne R: Viral miRNAs. Methods Mol Biol 721: 43-66, 2011.

22. Naito $\mathrm{Y}$ and Bono H: GGRNA: an ultrafast, transcript-oriented search engine for genes and transcripts. Nucleic Acids Res 40: W592-W596, 2012.

23. Cho WS: OncomiRs: the discovery and progress of microRNAs in cancers. Mol Cancer 6: 60, 2007.

24. Kong YW, Ferland-McCollough D, Jackson TJ and Bushell M: MicroRNAs in cancer management. Lancet Oncol 13: e249-e258, 2012.

25. Guo J, Miao Y, Xiao B, et al: Differential expression of microRNA species in human gastric cancer versus non-tumorous tissues. J Gastroenterol Hepatol 24: 652-657, 2009.

26. Lewis BP, Burge CB and Bartel DP: Conserved seed pairing, often flanked by adenosines, indicates that thousands of human genes are microRNA targets. Cell 120: 15-20, 2005.

27. Ott CE, Grünhagen J, Jäger M, et al: MicroRNAs differentially expressed in postnatal aortic development downregulate elastin via 3' UTR and coding-sequence binding sites. PLoS One 6: e16250, 2011.

28. Oh HJ, Chen X and Subjeck JR: Hsp110 protects heat-denatured proteins and confers cellular thermoresistance. J Biol Chem 272: 31636-31640, 1997.

29. Oh HJ, Easton D, Murawski M, Kaneko Y and Subjeck JR: The chaperoning activity of hsp110. Identification of functional domains by use of targeted deletions. J Biol Chem 274: 15712-15718, 1999.

30. Dorard C, de Thonel A, Collura A, et al: Expression of a mutant HSP110 sensitizes colorectal cancer cells to chemotherapy and improves disease prognosis. Nat Med 17: 1283-1289, 2011.

31. Den RB and Lu B: Heat shock protein 90 inhibition: rationale and clinical potential. Ther Adv Med Oncol 4: 211-218, 2012.

32. Bagatell R and Whitesell L: Altered Hsp90 function in cancer: a unique therapeutic opportunity. Mol Cancer Ther 3: 1021-1030, 2004.

33. Ito A, Saito H, Mitobe K, et al: Inhibition of heat shock protein 90 sensitizes melanoma cells to thermosensitive ferromagnetic particle-mediated hyperthermia with low Curie temperature. Cancer Sci 100: 558-564, 2009.

34. Workman P, Burrows F, Neckers L and Rosen N: Drugging the cancer chaperone HSP90: combinatorial therapeutic exploitation of oncogene addiction and tumor stress. Ann NY Acad Sci 1113: 202-216, 2007 